\title{
A FUNCTIONAL EQUATION CHARACTERIZING CUBIC POLYNOMIALS AND ITS STABILITY
}

\author{
SOON-MO JUNG and PRASANNA K. SAHOO
}

(Received 30 May 2000)

\begin{abstract}
We study the generalized Hyers-Ulam stability of the functional equation $f\left[x_{1}, x_{2}, x_{3}\right]=h\left(x_{1}+x_{2}+x_{3}\right)$.
\end{abstract}

2000 Mathematics Subject Classification. 39B22, 39B82.

1. Introduction. Given an operator $T$ and a solution class $\{u\}$ with the property that $T(u)=0$, when does $\|T(v)\| \leq \varepsilon$ for an $\varepsilon>0$ imply that $\|u-v\| \leq \delta(\varepsilon)$ for some $u$ and for some $\delta>0$ ? This problem is called the stability of the functional transformation. A great deal of work has been done in connection with the ordinary and partial differential equations. If $f$ is a function from a normed vector space into a Banach space, and $\|f(x+y)-f(x)-f(y)\| \leq \varepsilon$, Hyers [3] proved that there exists an additive map $A$ such that $\|f(x)-A(x)\| \leq \varepsilon$. If $f(x)$ is a real continuous function of $x$ over $\mathbb{R}$, and $|f(x+y)-f(x)-f(y)| \leq \varepsilon$, it was shown by Hyers and Ulam [4] that there exists a constant $k$ such that $|f(x)-k x| \leq 2 \varepsilon$. Taking these results into account, we say that the additive Cauchy equation $f(x+y)=f(x)+f(y)$ is stable in the sense of Hyers and Ulam.

In this paper, we study a generalized Hyers-Ulam stability of a mean value type functional equation.

Let $\mathbb{R}$ be the set of real numbers. For distinct points $x_{1}, x_{2}, \ldots, x_{n}$ in $\mathbb{R}$, the divided difference of $f: \mathbb{R} \rightarrow \mathbb{R}$ is recursively defined as

$$
\begin{gathered}
f\left[x_{1}\right]=f\left(x_{1}\right), \\
f\left[x_{1}, x_{2}, \ldots, x_{n}\right]=\frac{f\left[x_{1}, x_{2}, \ldots, x_{n-1}\right]-f\left[x_{2}, x_{3}, \ldots, x_{n}\right]}{x_{1}-x_{n}} .
\end{gathered}
$$

Bailey [2], generalizing a result of Aczel [1], proved the following result: if $f$ is $a$ differentiable function satisfying the functional equation

$$
f\left[x_{1}, x_{2}, x_{3}\right]=h\left(x_{1}+x_{2}+x_{3}\right), \quad \forall x_{1}, x_{2}, x_{3} \in \mathbb{R}
$$

with $x_{1} \neq x_{2}, x_{2} \neq x_{3}, x_{3} \neq x_{1}$, then $f$ is a polynomial of degree at most three. In Bailey's proof, the differentiability assumption plays a central role. Kannappan and Sahoo [5] have determined the general solution of $f\left[x_{1}, x_{2}, \ldots, x_{n}\right]=h\left(x_{1}+x_{2}+\cdots+\right.$ $x_{n}$ ) without the differentiability assumption. In the next section, we determine the general solution of (1.2) by an elementary method. 
2. Solution of the functional equation (1.2). Now we give the solution of the function equation (1.2) using an elementary technique.

THEOREM 2.1. Let $f$ satisfy the functional equation (1.2) for all $x_{1}, x_{2}, x_{3} \in \mathbb{R}$ with $x_{1} \neq x_{2}, x_{2} \neq x_{3}$, and $x_{3} \neq x_{1}$. Then $f$ is a polynomial of degree at most three and $h$ is linear.

Proof. If $f(x)$ is a solution of (1.2) so is $f(x)+a_{0}+a_{1} x$, where $a_{0}$ and $a_{1}$ are arbitrary constants. This can be verified by direct substitution into the expansion of the functional equation (1.2), that is,

$$
\begin{aligned}
\left(x_{2}-x_{3}\right) & f\left(x_{1}\right)+\left(x_{3}-x_{1}\right) f\left(x_{2}\right)+\left(x_{1}-x_{2}\right) f\left(x_{3}\right) \\
& =\left(x_{1}-x_{3}\right)\left(x_{1}-x_{2}\right)\left(x_{2}-x_{3}\right) h\left(x_{1}+x_{2}+x_{3}\right) .
\end{aligned}
$$

Letting $f\left(x_{i}\right)+a_{0}+a_{1} x_{i}$ for $i=1,2,3$ for $f\left(x_{i}\right)$ in the expansion (2.1), we get

$$
\begin{aligned}
& \left(x_{2}-x_{3}\right)\left[f\left(x_{1}\right)+a_{0}+a_{1} x_{1}\right]+\left(x_{3}-x_{1}\right)\left[f\left(x_{2}\right)+a_{0}+a_{1} x_{2}\right] \\
& \quad+\left(x_{1}-x_{2}\right)\left[f\left(x_{3}\right)+a_{0}+a_{1} x_{3}\right] \\
& =\left(x_{1}-x_{3}\right)\left(x_{1}-x_{2}\right)\left(x_{2}-x_{3}\right) h\left(x_{1}+x_{2}+x_{3}\right) .
\end{aligned}
$$

Each term involving an $a_{0}$ or an $a_{1}$ has an opposite-sign term and therefore cancels by simple algebraic manipulation. Thus we have again the expanded form (2.1) of (1.2). Let $g(x)=f(x)+a_{0}+a_{1} x$. Then $x=0$ inserted into $g(x)$ yields

$$
f(0)=g(0)-a_{0}
$$

We are free to pick $a_{0}=g(0)$ so that $g(x)$ yields $f(0)=0$. In other words, by a suitable choice for $a_{0}$, without loss of generality, we may assume that

$$
f(0)=0
$$

Now by setting $x=\alpha$ in the definition of $g(x)$ we get

$$
f(\alpha)=g(\alpha)-a_{0}-a_{1} \alpha
$$

Letting $a_{0}+a_{1} \alpha=g(\alpha)$ we get $f(\alpha)=0$ and we may assume, without loss of generality, that

$$
f(\alpha)=0
$$

for some $\alpha \neq 0$ in $\mathbb{R}$. Note that there are many choices for such an $\alpha$.

First substitute $(x, 0, \alpha)$ for $\left(x_{1}, x_{2}, x_{3}\right)$ in (2.1) to get

$$
f(x)=-x(\alpha-x) h(x+\alpha)
$$

(after using (2.4) and (2.6)) for $x \neq 0, \alpha$.

Next, we substitute $(x, 0, y)$ for $\left(x_{1}, x_{2}, x_{3}\right)$ in (2.1) to get

$$
\frac{f(x)}{x(x-y)}-\frac{f(y)}{y(x-y)}=h(x+y) \quad \forall x, y \neq 0, x \neq y .
$$


Define

$$
g(x)=\frac{f(x)}{x} \text { for } x \in \mathbb{R} \backslash\{0\}
$$

Then (2.8) reduces to

$$
g(x)-g(y)=(x-y) h(x+y) \quad \forall x, y \in \mathbb{R} \backslash\{0\} \text { with } x \neq y .
$$

Note that (2.10) is valid even for $x=y$.

Now we consider the equation

$$
g(x)-g(y)=(x-y) h(x+y) \quad \forall x, y \in \mathbb{R} \backslash\{0\} .
$$

Put $y=-x$ in (2.10) to get

$$
g(x)-g(-x)=2 x h(0) \quad \forall x \neq 0 .
$$

Next, replace $y$ by $-y$ in (2.10) to get

$$
g(x)-g(-y)=(x+y) h(x-y) \text { for } x, y \in \mathbb{R} \backslash\{0\} \text { with } x+y \neq 0 .
$$

Again (2.13) holds if $x+y=0$. Thus we conclude that (2.13) holds for $x, y \in \mathbb{R} \backslash\{0\}$. Subtract (2.10) from (2.13) and use (2.12) to get

$$
(x+y)[h(x-y)-h(0)]=(x-y)[h(x+y)-h(0)] \quad \forall x, y \in \mathbb{R} \backslash\{0\} .
$$

Fix a nonzero $u$ in $\mathbb{R}$. Choose a $v \in \mathbb{R}$ such that $(u+v) / 2 \neq 0$ and $(u-v) / 2 \neq 0$. There are plenty of choices for such $v$. Let

$$
x=\frac{u+v}{2}, \quad y=\frac{u-v}{2},
$$

so that

$$
u=x+y, \quad v=x-y .
$$

Letting (2.16) into (2.14), we get

$$
u[h(v)-h(0)]=v[h(u)-h(0)] \quad \forall v \neq u,-u .
$$

(Here note that $v$ can be zero since $x=y$ is allowed.) Hence for fixed $u=u_{1}$, we get

$$
h(v)=a_{1} v+b_{1} \quad \text { for } v \in \mathbb{R} \backslash\left\{u_{1},-u_{1}\right\} .
$$

Again $u=u_{2}$, we get

$$
h(v)=a_{2} v+b_{2} \quad \forall v \in \mathbb{R} \backslash\left\{u_{2},-u_{2}\right\} .
$$

Since the sets $\left\{u_{1},-u_{1}\right\}$ and $\left\{u_{2},-u_{2}\right\}$ are disjoint, we get

$$
h(v)=a v+b \quad \forall v \in \mathbb{R} .
$$

Now using (2.20) in (2.7), we have

$$
f(x)=\left(x^{2}-x \alpha\right) h(x+\alpha)=\left(x^{2}-x \alpha\right)[a(x+\alpha)+b]=a x^{3}+b x^{2}+c x,
$$


where $c=-a \alpha^{2}-b \alpha$. Removing the assumption that $f(0)=0$, we get

$$
f(x)=a x^{3}+b x^{2}+c x+d \quad \forall x \neq 0, \alpha .
$$

By (2.4), (2.6), and (2.22), we conclude that $f$ is a polynomial of degree at most three for all $x \in \mathbb{R}$. This proof is now complete.

For a more general result, the interested reader should refer to Kannappan and Sahoo [5].

3. Stability of the functional equation (1.2). Let $G$ be an additive subgroup of $\mathbb{C}$ and let $\varphi: G^{3} \rightarrow[0, \infty)$ be a control function. In the following theorem, the stability of (1.2) for cubic polynomials will be investigated in a modified form (3.1).

THEOREM 3.1. Let $\alpha \in G \backslash\{0\}$ and $\beta \in G \backslash\{-\alpha, 0, \alpha\}$ be fixed. If the functions $f, h: G \rightarrow \mathbb{C}$ satisfy the inequality

$$
\begin{aligned}
& \mid(y-z) f(x)+(z-x) f(y)+(x-y) f(z) \\
& \quad-(x-z)(x-y)(y-z) h(x+y+z) \mid \leq \varphi(x, y, z), \quad \forall x, y, z \in G,
\end{aligned}
$$

then there exist constants $a, b, c, d$ such that

$$
\begin{aligned}
\left|f(x)-a x^{3}-b x^{2}-c x-d\right| \leq & \frac{\left|x^{2}-\alpha^{2}\right|}{2|\beta|\left|\beta^{2}-\alpha^{2}\right|} \varphi(x, \beta,-\beta) \\
& +\frac{\left|x^{2}-\beta^{2}\right|}{2|\alpha|\left|\beta^{2}-\alpha^{2}\right|} \varphi(x, \alpha,-\alpha) \quad \forall x \in G, \\
|h(x)-a x-b| \leq & \frac{\left|x^{2}-\beta^{2}\right|+\left|\beta^{2}-\alpha^{2}\right|}{2|\alpha|\left|\beta^{2}-\alpha^{2}\right|\left|x^{2}-\alpha^{2}\right|} \varphi(x, \alpha,-\alpha) \\
& +\frac{1}{2|\beta|\left|\beta^{2}-\alpha^{2}\right|} \varphi(x, \beta,-\beta) \quad \forall x \in G \backslash\{-\alpha, \alpha\} .
\end{aligned}
$$

Moreover, the constants $a, b, c, d$ are explicitly given by

$$
\begin{aligned}
& a=\frac{f(\beta)-f(-\beta)}{2 \beta\left(\beta^{2}-\alpha^{2}\right)}-\frac{f(\alpha)-f(-\alpha)}{2 \alpha\left(\beta^{2}-\alpha^{2}\right)}, \\
& b=\frac{f(\beta)+f(-\beta)}{2\left(\beta^{2}-\alpha^{2}\right)}-\frac{f(\alpha)+f(-\alpha)}{2\left(\beta^{2}-\alpha^{2}\right)}, \\
& c=\frac{f(\alpha)-f(-\alpha)}{2 \alpha\left(\beta^{2}-\alpha^{2}\right)} \beta^{2}-\frac{f(\beta)-f(-\beta)}{2 \beta\left(\beta^{2}-\alpha^{2}\right)} \alpha^{2}, \\
& d=\frac{f(\alpha)+f(-\alpha)}{2\left(\beta^{2}-\alpha^{2}\right)} \beta^{2}-\frac{f(\beta)+f(-\beta)}{2\left(\beta^{2}-\alpha^{2}\right)} \alpha^{2} .
\end{aligned}
$$

Proof. If we define a function $g: G \rightarrow \mathbb{C}$ by

$$
g(x)=f(x)-\frac{f(\alpha)-f(-\alpha)}{2 \alpha} x-\frac{f(\alpha)+f(-\alpha)}{2},
$$


then $g(\alpha)=g(-\alpha)=0$ and $g$ satisfies the inequality

$$
\begin{aligned}
& \mid(y-z) g(x)+(z-x) g(y)+(x-y) g(z) \\
& \quad-(x-z)(x-y)(y-z) h(x+y+z) \mid \leq \varphi(x, y, z) \quad \forall x, y, z \in G .
\end{aligned}
$$

If we substitute $(x, \alpha,-\alpha)$ for $(x, y, z)$ in (3.6), then we have

$$
\left|g(x)-\left(x^{2}-\alpha^{2}\right) h(x)\right| \leq \frac{1}{2|\alpha|} \varphi(x, \alpha,-\alpha) \quad \forall x \text { in } G .
$$

Replace $z$ by $-y$ in (3.6) to get

$$
\left|2 y g(x)-(x+y) g(y)+(x-y) g(-y)-2 y\left(x^{2}-y^{2}\right) h(x)\right| \leq \varphi(x, y,-y)
$$

for every $x, y \in G$.

By making use of (3.7) and (3.8), we obtain

$$
\begin{aligned}
\left|2 y g(x)-(x+y) g(y)+(x-y) g(-y)-\frac{2 y\left(x^{2}-y^{2}\right)}{x^{2}-\alpha^{2}} g(x)\right| \\
\leq\left|2 y g(x)-(x+y) g(y)+(x-y) g(-y)-2 y\left(x^{2}-y^{2}\right) h(x)\right| \\
\quad+\left|2 y\left(x^{2}-y^{2}\right) h(x)-\frac{2 y\left(x^{2}-y^{2}\right)}{x^{2}-\alpha^{2}} g(x)\right| \\
\leq \varphi(x, y,-y)+\frac{|y|\left|x^{2}-y^{2}\right|}{|\alpha|\left|x^{2}-\alpha^{2}\right|} \varphi(x, \alpha,-\alpha)
\end{aligned}
$$

or equivalently

$$
\begin{aligned}
& \left|2 y \frac{y^{2}-\alpha^{2}}{x^{2}-\alpha^{2}} g(x)-(x+y) g(y)+(x-y) g(-y)\right| \\
& \quad \leq \varphi(x, y,-y)+\frac{|y|\left|x^{2}-y^{2}\right|}{|\alpha|\left|x^{2}-\alpha^{2}\right|} \varphi(x, \alpha,-\alpha), \quad \forall x \in G \backslash\{-\alpha, \alpha\}, \forall y \in G .
\end{aligned}
$$

Multiply both sides by

$$
\frac{\left|x^{2}-\alpha^{2}\right|}{2|y|\left|y^{2}-\alpha^{2}\right|}
$$

to get

$$
\begin{aligned}
\mid g(x) & -\frac{\left(x^{2}-\alpha^{2}\right)(x+y)}{2 y\left(y^{2}-\alpha^{2}\right)} g(y)+\frac{\left(x^{2}-\alpha^{2}\right)(x-y)}{2 y\left(y^{2}-\alpha^{2}\right)} g(-y) \mid \\
\leq & \frac{\left|x^{2}-\alpha^{2}\right|}{2|y|\left|y^{2}-\alpha^{2}\right|} \varphi(x, y,-y) \\
& +\frac{\left|x^{2}-y^{2}\right|}{2|\alpha|\left|y^{2}-\alpha^{2}\right|} \varphi(x, \alpha,-\alpha) \quad \forall x \in G, y \in G \backslash\{-\alpha, 0, \alpha\} .
\end{aligned}
$$

(We note that the inequality holds true also for $x \in\{-\alpha, \alpha\}$.)

If we replace $y$ in the last inequality by a constant $\beta \in G \backslash\{-\alpha, 0, \alpha\}$ and if we consider definition (3.5), then we can easily show the validity of inequality (3.2) by making a tedious calculation. 
By using (3.2), (3.5), and (3.7), we may obtain

$$
\begin{aligned}
& \left|\left(x^{2}-\alpha^{2}\right) h(x)-a x^{3}-b x^{2}+\alpha^{2} a x+\alpha^{2} b\right| \\
& \leq||\left(x^{2}-\alpha^{2}\right) h(x)-g(x) \mid \\
& \quad+\left|g(x)-f(x)+\frac{f(\alpha)-f(-\alpha)}{2 \alpha} x+\frac{f(\alpha)+f(-\alpha)}{2}\right| \\
& \quad+\left|f(x)-a x^{3}-b x^{2}-c x-d\right| \\
& \quad \leq \frac{\left|x^{2}-\beta^{2}\right|+\left|\beta^{2}-\alpha^{2}\right|}{2|\alpha|\left|\beta^{2}-\alpha^{2}\right|} \varphi(x, \alpha,-\alpha)+\frac{\left|x^{2}-\alpha^{2}\right|}{2|\beta|\left|\beta^{2}-\alpha^{2}\right|} \varphi(x, \beta,-\beta) \quad \forall x \in G,
\end{aligned}
$$

from which we can deduce inequality (3.3).

COROLlary 3.2. Assume that the control function $\varphi: G^{3} \rightarrow[0, \infty)$ satisfies the asymptotic condition

$$
\lim _{|x| \rightarrow \infty}|x|^{2} \varphi(x, \gamma,-\gamma)=0 \text { for each fixed } \gamma \in G .
$$

If the functions $f, h: G \rightarrow \mathbb{C}$ satisfy inequality (3.1) for any $x, y, z \in G$, then there exist uniquely determined constants $a, b, c, d$ such that inequalities (3.2) and (3.3) are valid for all $x \in G$ and for all $x \in G \backslash\{-\alpha, \alpha\}$, respectively.

COROLLARY 3.3. Suppose that the control function $\varphi: G^{3} \rightarrow[0, \infty)$ is given by

$$
\varphi(x, y, z)=\varepsilon|x-y||y-z||z-x| \text { for some given } \varepsilon>0 .
$$

If the functions $f, h: G \rightarrow \mathbb{C}$ satisfy inequality (3.1) for any $x, y, z \in G$, then there exist constants $a, b, c, d$ such that

$$
\begin{gathered}
\left|f(x)-a x^{3}-b x^{2}-c x-d\right| \leq \frac{2 \varepsilon}{\left|\beta^{2}-\alpha^{2}\right|}\left|x^{2}-\alpha^{2}\right|\left|x^{2}-\beta^{2}\right|, \\
|h(x)-a x-b| \leq \varepsilon+\frac{2 \varepsilon}{\left|\beta^{2}-\alpha^{2}\right|}\left|x^{2}-\beta^{2}\right| \quad \forall x \text { of } G .
\end{gathered}
$$

We remark here that the last inequality is also valid for $x=-\alpha$ or $x=\alpha$.

Given a control function $\psi: G^{3} \rightarrow[0, \infty)$, we can also prove the Hyers-Ulam-Rassias stability of the functional equation (1.2) in the original setting:

THEOREM 3.4. Let $\alpha \in G \backslash\{0\}$ and $\beta \in G \backslash\{-\alpha, 0, \alpha\}$ be given. If the functions $f, h: G \rightarrow \mathbb{C}$ satisfy the inequality

$$
|f[x, y, z]-h(x+y+z)| \leq \psi(x, y, z) \quad \forall x, y, z \in G \text { with } x \neq y, y \neq z, z \neq x,
$$

then there exist constants $a, b, c, d$ such that

$$
\begin{gathered}
\left|f(x)-a x^{3}-b x^{2}-c x-d\right| \leq \frac{\left|x^{2}-\alpha^{2}\right|\left|x^{2}-\beta^{2}\right|}{\left|\beta^{2}-\alpha^{2}\right|}(\psi(x, \alpha,-\alpha)+\psi(x, \beta,-\beta)), \\
|h(x)-a x-b| \leq \psi(x, \alpha,-\alpha)+\frac{\left|x^{2}-\beta^{2}\right|}{\left|\beta^{2}-\alpha^{2}\right|}(\psi(x, \alpha,-\alpha)+\psi(x, \beta,-\beta)),
\end{gathered}
$$

for all $x \in G$, where $a, b, c, d$ are explicitly given in Theorem 3.1. 
Proof. If we multiply both sides of (3.17) by $|x-y||y-z||z-x|$, then $f$ satisfies inequality (3.1) with

$$
\varphi(x, y, z)=|x-y||y-z||z-x| \psi(x, y, z) \quad \forall x, y, z \in G .
$$

(We note that (3.1) is also true for $x, y, z \in G$ with $x=y, y=z$, or $z=x$ for our case with (3.20).)

According to Theorem 3.1, there exist constants $a, b, c, d$ such that inequalities (3.18) and (3.19) are valid for all $x \in G$ and for all $x \in G \backslash\{-\alpha, \alpha\}$, respectively. The only reason for excepting $-\alpha$ and $\alpha$ from the domain of validity of inequality (3.3) is that the denominator of the first term on the right-hand side contains a factor $\left|x^{2}-\alpha^{2}\right|$. However, inequality (3.19) contains no denominator which vanishes at $x=\alpha$ or $x=-\alpha$. Therefore, we can include $-\alpha$ and $\alpha$ in the domain of validity of inequality (3.19), which completes the proof.

\section{REFERENCES}

[1] J. Aczél, A mean value property of the derivative of quadratic polynomials-without mean values and derivatives, Math. Mag. 58 (1985), no. 1, 42-45. MR 86c:39012. Zbl 571.39005.

[2] D. F. Bailey, A mean-value property of cubic polynomials - without mean values, Math. Mag. 65 (1992), no. 2, 123-124. CMP 1160 714. Zbl 786.39002.

[3] D. H. Hyers, On the stability of the linear functional equation, Proc. Nat. Acad. Sci. U.S.A. 27 (1941), 222-224. MR 2,315a. Zbl 061.26403.

[4] D. H. Hyers and S. M. Ulam, Approximately convex functions, Proc. Amer. Math. Soc. 3 (1952), 821-828. MR 14,254b. Zbl 047.29505.

[5] P. L. Kannappan and P. K. Sahoo, Characterization of polynomials and divided difference, Proc. Indian Acad. Sci. Math. Sci. 105 (1995), no. 3, 287-290. MR 97d:39010. Zbl 852.39005.

Soon-Mo Jung: Mathematics Section, College of Science \& Technology, Hong-IK UNIVERSITY, 339-701 CHOCHIWON, KOREA

E-mail address: smjung@wow.hongik.ac.kr

PRASANNA K. SAHOO: DEPARTMENT OF MATHEMATICS, UNIVERSITY OF LOUISVILLE, LOUISVILLE, KY 40292, USA

E-mail address: sahoo@1oui svi11e.edu 


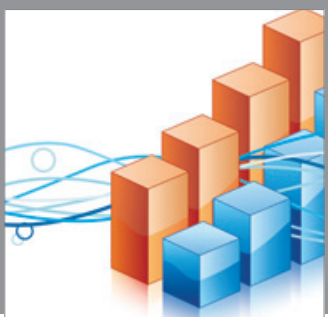

Advances in

Operations Research

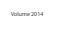

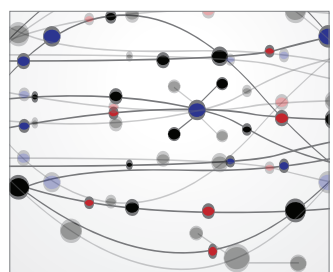

\section{The Scientific} World Journal
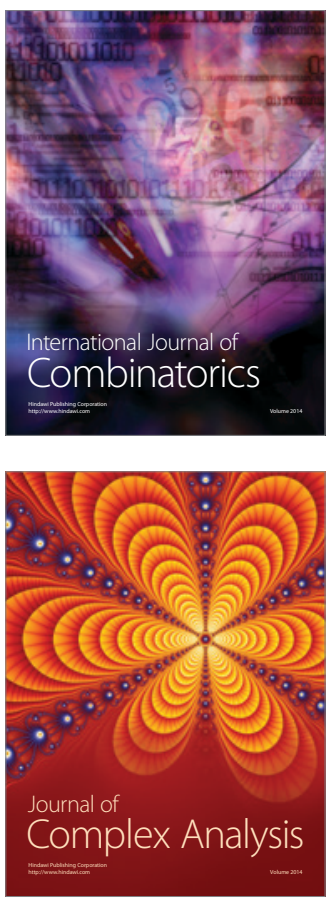

International Journal of

Mathematics and

Mathematical

Sciences
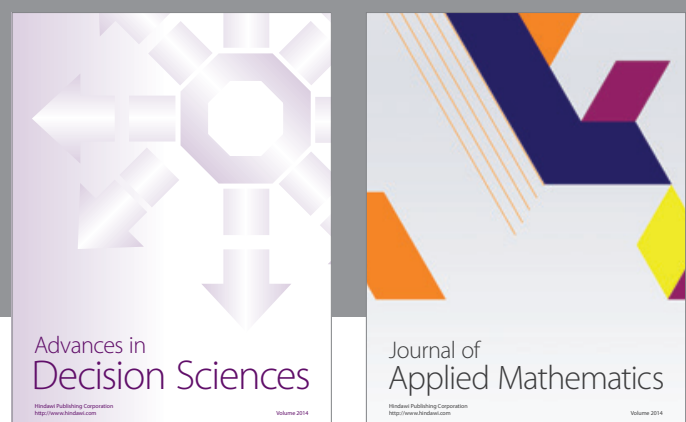

Journal of

Applied Mathematics
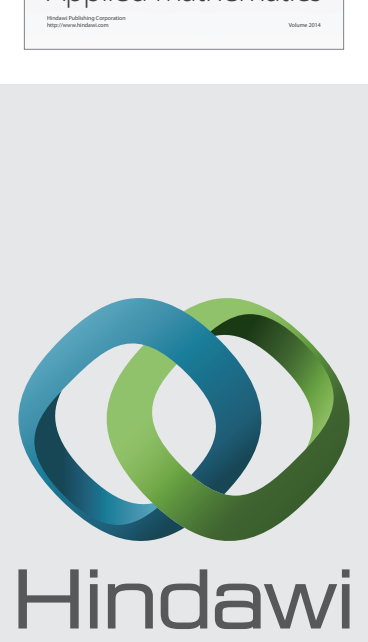

Submit your manuscripts at http://www.hindawi.com
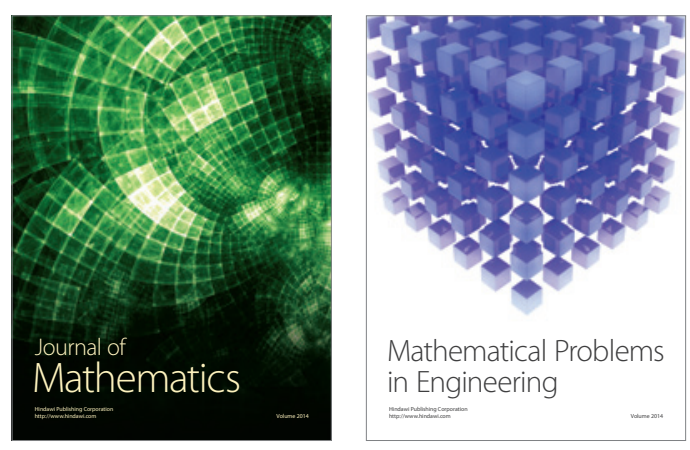

Mathematical Problems in Engineering
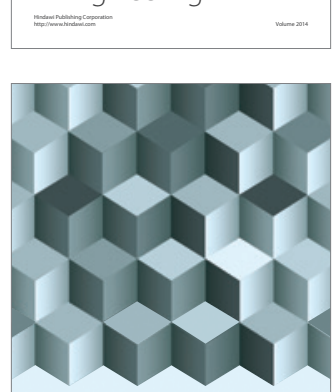

Journal of

Function Spaces
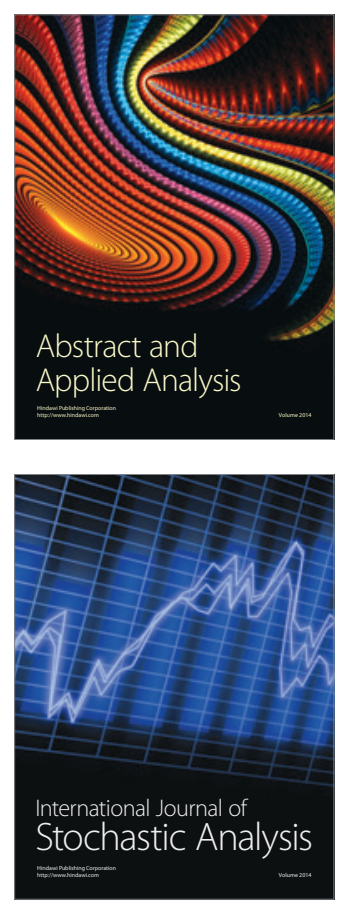

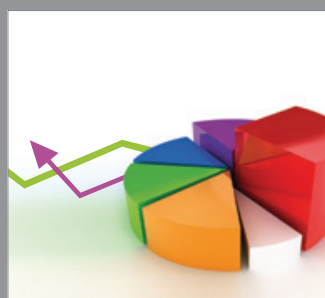

ournal of

Probability and Statistics

Promensencen
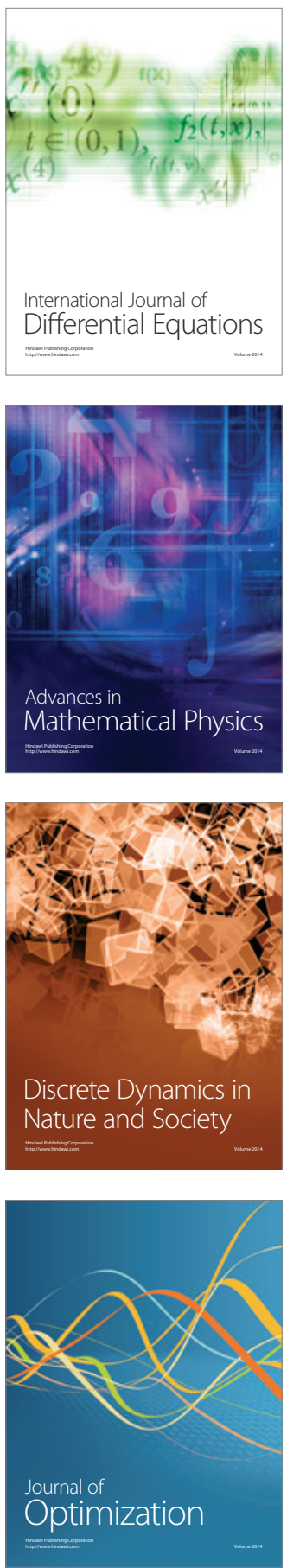